\title{
Medical costs and associated racial/ethnic and sex disparities in erythropoiesis-stimulating agent use: anemia management under the Medicare reimbursement policy
}

\author{
Minghui Li, PhD; Bo Cai, PhD; Patrick Mauldin, PhD; and Z Kevin Lu, PhD
}

\section{What is already known about this subject}

- Erythropoiesis-stimulating agents (ESAs) have an increased risk of death and were the highest-expenditure drug in the Medicare system before 2007.

- In 2007, CMS issued a Medicare reimbursement policy change for ESA use by cancer patients.

- The Medicare reimbursement policy change was effective in reducing the use of ESAs.

\section{What this study adds}

- Medical costs of ESAs used by cancer patients with chemotherapy-induced anemia were reduced after the 2007 Medicare reimbursement policy change.

- The policy change was only effective for patients who were male and those who were White.

- The policy change had no effect on patients who were female and those who were Black, Hispanic, and of other races and ethnicities.

\author{
Author affiliations \\ Minghui Li, PhD, Department of Clinical \\ Pharmacy and Translational Science, \\ University of Tennessee Health Science \\ Center, Memphis. Bo Cai, PhD, Department of \\ Epidemiology and Biostatistics, and Z Kevin \\ Lu, PhD, Department of Clinical Pharmacy \\ and Outcomes Sciences, University of South \\ Carolina, Columbia. Patrick Mauldin, PhD, \\ Department of Medicine, Medical University \\ of South Carolina, Charleston. \\ AUTHOR CORRESPONDENCE: \\ Z Kevin Lu, 803.777.2653; \\ lu32@email.sc.edu
}

$J$ Manag Care Spec Pharm 2021;27(8):1142-52

Copyright $\odot 2021$, Academy of Managed Care Pharmacy. All rights reserved.

on medical costs for cancer patients who were new users of ESAs.

METHODS: This study was an exploratory retrospective treatment effectiveness study, which used SEER-Medicare linked data. A difference-in-difference design was used that incorporated a control group of patients with chronic kidney disease. A generalized linear model, with a log link and a gamma distribution, was used to examine medical costs.

RESULTS: The Medicare reimbursement policy change was statistically significantly associated with an $11 \%(95 \% \mathrm{Cl}=2 \%-20 \%)$ reduction in anemia-related costs, including a $10 \%(95 \% \mathrm{Cl}=1 \%-19 \%)$ reduction in Medicare payment and an 18\% (95\% $\mathrm{Cl}=10 \%-26 \%)$ reduction in patient cost sharing. For total medical costs, the policy change was statistically significantly associated with a $12 \%(95 \% \mathrm{Cl}=6 \%-18 \%)$ reduction, including an $11 \%(95 \% \mathrm{Cl}=5 \%-18 \%)$ reduction in Medicare payment and a $14 \%$ (95\% $\mathrm{Cl}=7 \%-20 \%$ ) reduction in patient cost sharing. Medical costs were reduced in patients who were male and those who were White but remained the same for patients who were female and those who were Black, Hispanic, and other races or ethnicities. 
CONCLUSIONS: Anemia-related and total medical costs associated with ESAs used by cancer patients with chemotherapy-induced anemia were reduced after the Medicare reimbursement policy change. However, the policy change was only effective for patients who were male and those who were White. The policy change had no effect on patients who were female and those of racial/ethnic minorities.

Anemia is a prevalent complication of cancer. About 30\%$90 \%$ of cancer patients also have anemia. ${ }^{1}$ The prevalence of anemia varies by cancer type and disease stage and is associated with reduced quality of life in cancer patients. ${ }^{2}$ Myelosuppressive chemotherapy could impair hematopoiesis in the bone marrow and decrease the production of erythropoietin in the renal cortex. ${ }^{3}$ The type of malignancy is associated with the incidence and severity of chemotherapy-induced anemia. ${ }^{4}$ Patients with lung tumors, gynecologic tumors, genitourinary tumors, lymphomas, and colorectal tumors have a high incidence of chemotherapy-induced anemia. ${ }^{2,3}$

The most effective pharmacological treatment for anemia in cancer patients is an erythropoiesis-stimulating agent (ESA), such as epoetin and darbepoetin. Before 2007, ESAs were the highest-expenditure drug in the Medicare system, accounting for $\$ 2$ billion in Medicare Part B. ${ }^{6}$ The appropriate use of ESAs has been recommended in the American Society of Clinical Oncology (ASCO) and the American Society of Hematology (ASH) clinical practice guidelines in 2002, 2007, and 2010.-9 The ASCO/ASH guidelines recommend initiating ESAs in cancer patients with a hemoglobin level less than $10 \mathrm{~g} / \mathrm{dL}$. It is uncertain whether ESAs should be initiated when hemoglobin levels are between $10 \mathrm{~g} / \mathrm{dL}$ and $12 \mathrm{~g} / \mathrm{dL}$. The recommended hemoglobin initiation levels were consistent across different guideline updates.

Because of the increased risk of death, myocardial infarction, stroke, venous thromboembolism, and tumor progression or recurrence associated with inappropriate use of ESAs, in 2007 the Centers for Medicare \& Medicaid Services (CMS) issued a reimbursement policy change for the use of ESAs in cancer and related neoplastic conditions..$^{10-13}$ Compared with recommendations in the ASCO/ ASH guidelines, CMS has had more rigid requirements for ESA use. When ESAs have been used to treat chemotherapy-induced anemia in patients with solid tumors, multiple myeloma, lymphoma, or lymphocytic leukemia, Medicare has denied payment of services if the hemoglobin level is $10 \mathrm{~g} / \mathrm{dL}$ or higher. Patients who qualify for the reasonable and necessary use of ESAs could still have access to ESA treatment. However, providers and dispensers required the signing of an Advance Beneficiary Notice of Noncoverage so that patients were fully liable for payment of services not covered under Medicare. The monthly patient costs for ESAs were about $\$ 1,000$ when not covered. ${ }^{14}$

The use of ESAs increased 3-fold between the US Food and Drug Administration's (FDA) approval of darbepoetin in 2001 and the implementation of the Medicare reimbursement policy. ${ }^{15}$ After the policy change, a $26 \%-57 \%$ reduction in ESA use was documented in the literature. Hess et al and Henry et al found that the use of ESAs had a 29\%$36 \%$ reduction after the policy change based on medical records. ${ }^{16,17}$ Arneson et al, Hershman et al, and Li et al found that the use of ESAs had a 50\%-57\% reduction after the policy change based on claims. ${ }^{18-20}$ Specifically, Li et al identified racial/ethnic and sex disparities in the impact of the policy change on the use of ESAs..$^{20}$

Before the reimbursement policy change, Medicare paid approximately $\$ 2$ billion every year to reimburse for ESAs. ${ }^{21}$ To the best of our knowledge, no studies have examined the impact of the Medicare reimbursement policy change on medical costs associated with ESAs. After the policy change, adverse events associated with ESAs could have decreased, which would have resulted in a reduction of total medical costs. Because of racial/ethnic and sex disparities in the use of ESAs, the policy change could have had different effects on different groups in terms of medical costs. In the United States, CMS is the largest health care payer. Because ESAs were the highest-expenditure drug in Medicare Part B before the policy change, medical costs are of great interest to CMS. However, empirical evidence is currently lacking to evaluate medical costs under the policy, especially by sex and racial/ethnic groups.

This study fills the gap in the literature by evaluating the impact of the Medicare reimbursement policy change on medical costs associated with ESAs. The objective of this study was to examine the impact of the Medicare reimbursement policy change on medical costs in cancer patients, along with associated racial/ethnic and sex disparities.

\section{Methods}

\section{DATA SOURCE}

Surveillance, Epidemiology, and End Results (SEER)Medicare linked data were used in this study. SEER includes several population-based cancer registries across the United States. Medicare is a US national health insurance program. The linked data were appropriate for this study because they contain information on demographics, cancer incidence and mortality, health service utilization, 
diagnosis, and payments for Medicare beneficiaries with cancer. The SEER-Medicare data include medical costs as study outcomes and demographics, socioeconomics, and clinical characteristics of cancer patients as covariates. The SEER-Medicare linked data also contain a noncancer cohort for comparison purposes.

\section{STUDY DESIGN}

This study was an exploratory retrospective treatment effectiveness study that used a retrospective incident user cohort design. The index date for the incident user of ESAs was the date of the first ESA prescription. The cohort design enabled us to estimate anemia-related and total medical costs since the initiation of an ESA. The Medicare reimbursement policy change regarding ESAs became effective in July 2007 and was fully implemented in April 2008. Individuals who initiated ESAs between May 2005 and December 2006 were considered as incident users in the prepolicy period; individuals who initiated ESAs between May 2008 and December 2009 were considered as incident users in the postpolicy period. We selected the postpolicy period based on the timeline of the Medicare reimbursement policy change and then selected the corresponding prepolicy period to minimize the impact on the implementation of the policy change on study outcomes.

\section{CONTROL GROUP}

This study incorporated a control group of patients with chronic kidney disease (CKD). The FDA had a black-box warning about the increased risk of ESAs in patients with cancer or CKD. In the labeling of ESAs, initiation is appropriate only if the hemoglobin level is less than $10 \mathrm{~g} / \mathrm{dL}$. The labeling, however, does not specify at which hemoglobin level the ESA treatment should be suspended. The Kidney Disease Outcomes Quality Initiative (KDOQI) published clinical practice guidelines on the hemoglobin target for anemia in CKD in 2007.22 The KDOQI guidelines recommended considering potential benefits and harms when initiating ESAs. The specific hemoglobin threshold with which to initiate ESAs was not mentioned in the KDOQI guidelines. The Medicare reimbursement policy change regarding ESA use applied to cancer patients but did not apply to CKD patients, whereas the FDA's black box warning on ESAs applied to cancer and CKD.

\section{STUDY POPULATION}

The study population of the treatment group was selected from individuals who were aged 66 years or older; were eligible for Medicare because of age; had a primary diagnosis of breast cancer, colorectal cancer, lung cancer, lymphomas, ovarian cancer, or prostate cancer; received chemotherapy after a cancer diagnosis; initiated an ESA after chemotherapy; and initiated an ESA during the study period. This study included breast cancer, colorectal cancer, lung cancer, lymphomas, ovarian cancer, and prostate cancer because of the high incidence of chemotherapyinduced anemia. Excluded from the treatment group were individuals who enrolled in health maintenance organization (HMO) plans that did not have coverage for Medicare Parts A and B, were eligible for Medicare because of endstage renal disease (ESRD), had a diagnosis of CKD, or received an ESA 1 year before the index date.

The study population of the control group was selected from individuals who were aged 66 years or older, were eligible for Medicare because of ESRD or had a diagnosis of CKD, initiated an ESA after CKD diagnosis, and initiated an ESA during the study period. Individuals who enrolled in HMO plans, did not have coverage for Medicare Parts A and $\mathrm{B}$, had a diagnosis of cancer, or received an ESA 1 year before the index date were excluded from the control group.

\section{MEASUREMENT}

Medical costs were measured from the perspective of CMS and from the patient perspective. To measure anemiarelated and total medical costs, incident users of ESAs were followed up for 1 year. During this follow-up period, medical costs were measured based on payment information from Medicare claims, which included Medicare provider, carrier, outpatient, home health agency, hospice, and durable medical equipment. Anemia-related costs included costs of ESAs, blood transfusion, granulocytecolony stimulating factor, and other anemia treatments as recorded in Medicare claims.

Total medical costs included all costs as recorded in Medicare claims. Anemia-related and total medical costs comprised Medicare reimbursement and patient cost sharing. The Consumer Price Index, with the medical care component, was used to calculate the inflation rate and adjust medical costs that occurred in different years to 2010 prices. ESAs were measured based on Healthcare Common Procedure Coding System/Current Procedural Terminology and revenue center codes.

Covariates considered were demographics (age, sex, and race); socioeconomics (urbanicity, region, education level, and poverty level); and clinical characteristics (comorbidity and vital status). They were measured based on the SEER registries and Medicare enrollment and claims.

\section{STATISTICAL ANALYSIS}

Chi-square tests were used to compare baseline characteristics of incident users of ESAs before and after the Medicare reimbursement policy change. Independent sample t-tests 


\section{TABLE 1 Baseline Characteristics of Incident ESA Users Between Pre- and Postpolicy Periods} by Treatment Group

\begin{tabular}{|c|c|c|c|c|c|c|}
\hline & \multicolumn{3}{|c|}{ Treatment group $(n=17,382)$} & \multicolumn{3}{|c|}{ Control group $(n=3,069)$} \\
\hline & $\begin{array}{c}\text { Prepolicy } \\
(n=12,892), \%\end{array}$ & $\begin{array}{l}\text { Postpolicy } \\
(n=4,490), \%\end{array}$ & $P$ value & $\begin{array}{c}\text { Prepolicy } \\
(n=1,763), \%\end{array}$ & $\begin{array}{l}\text { Postpolicy } \\
(n=1,306), \%\end{array}$ & $P$ value \\
\hline \multicolumn{7}{|l|}{ Age, years } \\
\hline $66-69$ & 26.72 & 25.75 & \multirow{4}{*}{0.1606} & 14.97 & 15.16 & \multirow{4}{*}{0.3033} \\
\hline $70-74$ & 29.94 & 29.82 & & 18.49 & 17.92 & \\
\hline $75-79$ & 23.95 & 25.55 & & 24.33 & 21.82 & \\
\hline $80+$ & 19.39 & 18.89 & & 42.20 & 45.10 & \\
\hline \multicolumn{7}{|l|}{ Sex } \\
\hline Male & 39.87 & 40.33 & \multirow{2}{*}{0.5843} & 45.09 & 43.87 & \multirow{2}{*}{0.5017} \\
\hline Female & 60.13 & 59.67 & & 54.91 & 56.13 & \\
\hline \multicolumn{7}{|l|}{ Race/ethnicity } \\
\hline White & 88.37 & 86.70 & \multirow{4}{*}{0.0150} & 75.53 & 74.00 & \multirow{4}{*}{0.2029} \\
\hline Black & 6.65 & 7.22 & & 13.57 & 14.65 & \\
\hline Hispanic & 0.93 & 1.09 & & 3.07 & 4.29 & \\
\hline Other & 4.04 & 4.99 & & 7.84 & 7.06 & \\
\hline \multicolumn{7}{|l|}{ Urbanicity } \\
\hline Metropolitan & 83.20 & 80.96 & \multirow{2}{*}{0.0007} & 85.41 & 82.91 & \multirow{2}{*}{0.0593} \\
\hline Nonmetropolitan & 16.80 & 19.04 & & 14.59 & 17.09 & \\
\hline \multicolumn{7}{|l|}{ Region } \\
\hline Northeast & 21.68 & 20.76 & \multirow{4}{*}{0.0017} & 22.12 & 18.15 & \multirow{4}{*}{0.0293} \\
\hline Midwest & 11.98 & 11.43 & & 11.51 & 10.87 & \\
\hline South & 27.54 & 30.58 & & 27.96 & 31.09 & \\
\hline West & 38.79 & 37.24 & & 38.40 & 39.89 & \\
\hline \multicolumn{7}{|l|}{ Education $^{a}$} \\
\hline 1st quartile & 25.37 & 24.03 & \multirow{4}{*}{0.0055} & 21.77 & 19.94 & \multirow{4}{*}{0.5052} \\
\hline 2nd quartile & 25.28 & 23.91 & & 22.47 & 24.00 & \\
\hline 3rd quartile & 25.23 & 25.42 & & 25.10 & 24.32 & \\
\hline 4th quartile & 24.13 & 26.64 & & 30.66 & 31.74 & \\
\hline
\end{tabular}

continued on next page

were used to compare average medical costs in incident users of ESAs between the pre- and postpolicy periods. A difference-in-difference design was used to examine the impact of the Medicare reimbursement policy change on anemia-related and total medical costs associated with ESAs. ${ }^{23}$ A generalized linear model, with a log link and a gamma distribution, was used in the difference-in-difference design. ${ }^{24}$ The interaction term between the policy change and group assignment was the key variable of interest. Subgroup analyses were conducted by each sex and racial/ethnic group (male, female, White, Black, Hispanic, and other races/ethnicities) to examine associated disparities. All statistical analyses were conducted using SAS version 9.2 (SAS Institute).

\section{Results}

In the treatment group, we identified 17,382 incident ESA users, including 12,892 (74.17\%) in the prepolicy period and $4,490(25.83 \%)$ in the postpolicy period. In the control group, we identified 3,069 incident ESA users, including $1,763(57.45 \%)$ in the prepolicy period and 1,306 (42.55\%) 


\section{TABLE 1 Baseline Characteristics of Incident ESA Users Between Pre- and Postpolicy Periods by Treatment Group (continued)}

\begin{tabular}{|c|c|c|c|c|c|c|}
\hline & \multicolumn{3}{|c|}{ Treatment group $(n=17,382)$} & \multicolumn{3}{|c|}{ Control group $(n=3,069)$} \\
\hline & $\begin{array}{c}\text { Prepolicy } \\
(n=12,892), \%\end{array}$ & $\begin{array}{c}\text { Postpolicy } \\
(n=4,490), \%\end{array}$ & $P$ value & $\begin{array}{c}\text { Prepolicy } \\
(n=1,763), \%\end{array}$ & $\begin{array}{c}\text { Postpolicy } \\
(n=1,306), \%\end{array}$ & $P$ value \\
\hline \multicolumn{7}{|l|}{ Poverty ${ }^{b}$} \\
\hline 1st quartile & 25.43 & 24.59 & \multirow{4}{*}{0.0242} & 22.76 & 20.10 & \multirow{4}{*}{0.3630} \\
\hline 2nd quartile & 25.45 & 23.82 & & 23.23 & 23.92 & \\
\hline 3rd quartile & 24.72 & 25.23 & & 23.99 & 24.32 & \\
\hline 4th quartile & 24.40 & 26.36 & & 30.02 & 31.66 & \\
\hline \multicolumn{7}{|l|}{$\mathrm{CCl}$ score } \\
\hline 0 & 50.16 & 43.96 & \multirow{4}{*}{$<0.0001$} & 21.10 & 19.60 & \multirow{4}{*}{0.3625} \\
\hline 1 & 31.61 & 35.19 & & 23.37 & 25.34 & \\
\hline 2 & 12.46 & 13.41 & & 23.60 & 21.98 & \\
\hline $3+$ & 5.77 & 7.44 & & 31.93 & 33.08 & \\
\hline \multicolumn{7}{|c|}{ Vital status during the follow-up period } \\
\hline Alive & 56.14 & 51.94 & \multirow{2}{*}{$<0.0001$} & 76.97 & 76.72 & \multirow{2}{*}{0.8719} \\
\hline Dead & 43.86 & 48.06 & & 23.03 & 23.28 & \\
\hline Anemia-related costs, mean (SD) & $\begin{array}{c}\$ 8,153.19 \\
(\$ 10,391.06)\end{array}$ & $\begin{array}{c}\$ 7,843.67 \\
(\$ 13,509.89)\end{array}$ & 0.1622 & $\begin{array}{c}\$ 8,740.53 \\
(\$ 13,761.47)\end{array}$ & $\begin{array}{c}\$ 9,282.03 \\
(\$ 14,599.46)\end{array}$ & 0.2980 \\
\hline Medicare payment & $\begin{array}{c}\$ 6,794.42 \\
(\$ 9,237.05)\end{array}$ & $\begin{array}{c}\$ 6,829.34 \\
(\$ 12,544.48)\end{array}$ & 0.8642 & $\begin{array}{c}\$ 7,431.68 \\
(\$ 12,424.46)\end{array}$ & $\begin{array}{c}\$ 8,084.79 \\
(\$ 13,440.04)\end{array}$ & 0.1695 \\
\hline Patient cost sharing & $\begin{array}{c}\$ 1,358.76 \\
(\$ 1,644.45)\end{array}$ & $\begin{array}{c}\$ 1,014.33 \\
(\$ 1,442.35)\end{array}$ & $<0.0001$ & $\begin{array}{c}\$ 1,308.85 \\
(\$ 1,768.06)\end{array}$ & $\begin{array}{c}\$ 1,197.24 \\
(\$ 1,768.55)\end{array}$ & 0.0839 \\
\hline Total medical costs, mean (SD) & $\begin{array}{c}\$ 58,777.49 \\
(\$ 41,369.36)\end{array}$ & $\begin{array}{c}\$ 55,850.92 \\
(\$ 40,494.11)\end{array}$ & $<0.0001$ & $\begin{array}{c}\$ 51,476.36 \\
(\$ 53,823.64)\end{array}$ & $\begin{array}{c}\$ 55,219.84 \\
(\$ 58,325.85)\end{array}$ & 0.0694 \\
\hline Medicare payment & $\begin{array}{c}\$ 48,845.85 \\
(\$ 35,429.73)\end{array}$ & $\begin{array}{c}\$ 46,921.85 \\
(\$ 34,962.77)\end{array}$ & 0.0017 & $\begin{array}{c}\$ 44,260.17 \\
(\$ 47,398.36)\end{array}$ & $\begin{array}{c}\$ 47,602.76 \\
(\$ 50,850.23)\end{array}$ & 0.0640 \\
\hline Patient cost sharing & $\begin{array}{c}\$ 9,931.64 \\
(\$ 7,365.37)\end{array}$ & $\begin{array}{c}\$ 8,929.06 \\
(\$ 6,706.04)\end{array}$ & $<0.0001$ & $\begin{array}{c}\$ 7,216.19 \\
(\$ 7,440.38)\end{array}$ & $\begin{array}{c}\$ 7,617.09 \\
(\$ 8,493.59)\end{array}$ & 0.1733 \\
\hline
\end{tabular}

aPercentage of persons aged 25 years or older with less than 12 years of education.

${ }^{b}$ Percentage of population below poverty level.

$\mathrm{CCl}=$ Charlson Comorbidity Index; ESA = erythropoiesis-stimulating agent.

in the postpolicy period (Table 1). Except for age and sex, all other baseline characteristics were statistically significantly different in incident users of ESA between the pre-and postpolicy periods in the treatment group. Except for region, all other baseline characteristics were similar for ESA incident users between the pre- and postpolicy periods in the control group (Table 1).

For anemia-related costs, the Medicare reimbursement policy change was statistically significantly associated with an $11 \%(95 \% \mathrm{CI}=2 \%-20 \%)$ reduction after controlling for potential confounding factors (Table 2). Other factors statistically significantly associated with the change in anemia-related costs were age, sex, race, urbanicity, region, education, poverty, comorbidity, and vital status. When examining Medicare payment of anemia-related costs, we found that the Medicare reimbursement policy change was statistically significantly associated with a $10 \%(95 \%$ $\mathrm{CI}=1 \%-19 \%)$ reduction in Medicare payment and an $18 \%$ (95\% CI $=10 \%-26 \%)$ reduction in patient cost sharing of anemia-related costs (Table 2).

For total medical costs, the Medicare reimbursement policy change was statistically significantly associated with a $12 \%(95 \% \mathrm{CI}=6 \%-18 \%)$ reduction after controlling for potential confounding factors (Table 3). Other factors 


\section{TABLE 2 Change in Anemia-Related Costs Before and After Medicare Reimbursement Policy Change}

\begin{tabular}{|c|c|c|c|c|c|c|}
\hline & \multicolumn{2}{|c|}{ Anemia-related costs } & \multicolumn{2}{|c|}{$\begin{array}{l}\text { Medicare payment of } \\
\text { anemia-related costs }\end{array}$} & \multicolumn{2}{|c|}{$\begin{array}{l}\text { Patient cost sharing of } \\
\text { anemia-related costs }\end{array}$} \\
\hline & $\beta$ & $95 \% \mathrm{Cl}$ & $\beta$ & $95 \% \mathrm{Cl}$ & $\beta$ & $95 \% \mathrm{Cl}$ \\
\hline \multicolumn{7}{|l|}{ Policy change } \\
\hline Prepolicy period & Ref & & Ref & & Ref & \\
\hline Postpolicy period & 0.05 & $(-0.03,0.13)$ & 0.07 & $(-0.01,0.15)$ & -0.09 & $(-0.16,-0.02)$ \\
\hline \multicolumn{7}{|l|}{ Group assignment } \\
\hline Control group & Ref & & Ref & & Ref & \\
\hline Treatment group & -0.07 & $(-0.13,-0.01)$ & -0.09 & $(-0.15,-0.03)$ & 0.05 & $(0.00,0.10)$ \\
\hline \multicolumn{7}{|l|}{ Policy change $\times$ group assignment } \\
\hline Otherwise & Ref & & Ref & & Ref & \\
\hline Postpolicy period and treatment group & -0.11 & $(-0.20,-0.02)$ & -0.10 & $(-0.19,-0.01)$ & -0.18 & $(-0.26,-0.10)$ \\
\hline \multicolumn{7}{|l|}{ Age, years } \\
\hline $66-69$ & Ref & & Ref & & Ref & \\
\hline $70-74$ & -0.08 & $(-0.12,-0.04)$ & -0.09 & $(-0.13,-0.04)$ & -0.04 & $(-0.08,0.00)$ \\
\hline $75-79$ & -0.08 & $(-0.12,-0.03)$ & -0.09 & $(-0.13,-0.04)$ & -0.04 & $(-0.08,0.00)$ \\
\hline $80+$ & -0.17 & $(-0.21,-0.13)$ & -0.18 & $(-0.22,-0.13)$ & -0.14 & $(-0.18,-0.10)$ \\
\hline \multicolumn{7}{|l|}{ Sex } \\
\hline Male & 0.09 & $(0.05,0.12)$ & 0.09 & $(0.06,0.12)$ & 0.08 & $(0.06,0.11)$ \\
\hline Female & Ref & & Ref & & Ref & \\
\hline \multicolumn{7}{|l|}{ Race/ethnicity } \\
\hline White & Ref & & Ref & & Ref & \\
\hline Black & 0.07 & $(0.01,0.13)$ & 0.07 & $(0.01,0.13)$ & 0.04 & $(-0.02,0.09)$ \\
\hline Hispanic & 0.04 & $(-0.09,0.18)$ & 0.06 & $(-0.08,0.20)$ & -0.08 & $(-0.20,0.05)$ \\
\hline Other & -0.04 & $(-0.11,0.04)$ & -0.03 & $(-0.10,0.05)$ & -0.07 & $(-0.14,0.00)$ \\
\hline \multicolumn{7}{|l|}{ Urbanicity } \\
\hline Metropolitan & Ref & & Ref & & Ref & \\
\hline Nonmetropolitan & -0.07 & $(-0.11,-0.02)$ & -0.08 & $(-0.13,-0.03)$ & 0.02 & $(-0.03,0.06)$ \\
\hline
\end{tabular}

statistically significantly associated with the change in total medical costs included age, sex, race, urbanicity, region, education, poverty, comorbidity, and vital status. When examining Medicare payment of total medical costs, we found that the Medicare reimbursement policy change was statistically significantly associated with an $11 \%(95 \%$ $\mathrm{CI}=5 \%-18 \%)$ reduction in Medicare payment and a $14 \%$ $(95 \% \mathrm{CI}=7 \%-20 \%)$ reduction in patient cost sharing of total medical costs (Table 3).

The Medicare reimbursement policy change had different effects on economic outcomes of different sex and racial/ethnic groups. Anemia-related costs were reduced by $21 \%$ for patients who were male $(P=0.0019)$ and $16 \%$ for patients who were White $(P=0.0011)$ but remained the same for those who were female $(\beta=-0.04, P=0.4504)$, Black $(\beta=0.12, P=0.3770)$, Hispanic $(\beta=0.27, P=0.3830)$, or other races/ethnicities $(\beta=-0.06, P=0.7338)$ after the policy change. Total medical costs were reduced by $18 \%$ for patients who were male $(P=0.0001)$ and $16 \%$ for patients who were White $(P<0.0001)$ but remained the same for those who were female $(\beta=-0.08, P=0.0628)$, Black $(\beta=0.10$, $P=0.3278)$, Hispanic $(\beta=-0.10, P=0.6502)$, or other races/ ethnicities $(\beta=-0.04, P=0.7613)$ after the policy change. Patient cost sharing was reduced after the policy change for patients who were male and female (Table 4). 


\section{TABLE 2 Change in Anemia-Related Costs Before and After Medicare Reimbursement Policy Change (continued)}

\begin{tabular}{|c|c|c|c|c|c|c|}
\hline & \multicolumn{2}{|c|}{ Anemia-related costs } & \multicolumn{2}{|c|}{$\begin{array}{l}\text { Medicare payment of } \\
\text { anemia-related costs }\end{array}$} & \multicolumn{2}{|c|}{$\begin{array}{l}\text { Patient cost sharing of } \\
\text { anemia-related costs }\end{array}$} \\
\hline & $\beta$ & $95 \% \mathrm{Cl}$ & $\beta$ & $95 \% \mathrm{Cl}$ & $\beta$ & $95 \% \mathrm{Cl}$ \\
\hline \multicolumn{7}{|l|}{ Region } \\
\hline Northeast & 0.05 & $(0.00,0.09)$ & 0.04 & $(-0.01,0.09)$ & 0.10 & $(0.06,0.14)$ \\
\hline Midwest & 0.00 & $(-0.06,0.05)$ & -0.02 & $(-0.07,0.04)$ & 0.06 & $(0.01,0.11)$ \\
\hline South & -0.04 & $(-0.09,0.00)$ & -0.06 & $(-0.10,-0.02)$ & 0.06 & $(0.02,0.10)$ \\
\hline West & Ref & & Ref & & Ref & \\
\hline \multicolumn{7}{|l|}{ Education $^{\mathrm{a}}$} \\
\hline 1st quartile & Ref & & Ref & & Ref & \\
\hline 2nd quartile & -0.01 & $(-0.05,0.04)$ & 0.00 & $(-0.05,0.04)$ & -0.01 & $(-0.05,0.03)$ \\
\hline 3rd quartile & 0.07 & $(0.01,0.12)$ & 0.07 & $(0.02,0.13)$ & 0.04 & $(-0.01,0.09)$ \\
\hline 4th quartile & 0.08 & $(0.01,0.14)$ & 0.09 & $(0.03,0.16)$ & -0.03 & \\
\hline \multicolumn{7}{|l|}{ Poverty ${ }^{b}$} \\
\hline 1st quartile & Ref & & Ref & & Ref & \\
\hline 2nd quartile & -0.04 & $(-0.09,0.01)$ & -0.05 & $(-0.10,0.00)$ & -0.02 & $(-0.06,0.03)$ \\
\hline 3rd quartile & -0.06 & $(-0.11,0.00)$ & -0.07 & $(-0.13,-0.01)$ & 0.02 & $(-0.03,0.07)$ \\
\hline 4th quartile & -0.06 & $(-0.13,0.01)$ & -0.08 & $(-0.15,-0.01)$ & 0.01 & $(-0.06,0.07)$ \\
\hline \multicolumn{7}{|l|}{$\mathrm{CCl}$ score } \\
\hline 0 & Ref & & Ref & & Ref & \\
\hline 1 & 0.04 & $(0.00,0.07)$ & 0.04 & $(0.00,0.08)$ & 0.03 & $(0.00,0.06)$ \\
\hline 2 & 0.08 & $(0.04,0.13)$ & 0.10 & $(0.05,0.15)$ & 0.01 & $(-0.04,0.05)$ \\
\hline $3+$ & 0.15 & $(0.09,0.21)$ & 0.16 & $(0.10,0.22)$ & 0.09 & $(0.03,0.14)$ \\
\hline \multicolumn{7}{|c|}{ Vital status during the follow-up period } \\
\hline Alive & Ref & & Ref & & Ref & \\
\hline Dead & 0.16 & $(0.12,0.19)$ & 0.20 & $(0.16,0.23)$ & -0.08 & $(-0.11,-0.05)$ \\
\hline
\end{tabular}

apercentage of persons aged 25 years or older with less than 12 years of education.

${ }^{b}$ Percentage of the population below poverty level.

$\mathrm{CCl}=$ Charlson Comorbidity Index; Ref = reference.

\section{Discussion}

Based on the current evidence in the literature, among ESA users, it was not clear as to how anemia-related and total medical costs would change after the implementation of the Medicare reimbursement policy, especially by sex and racial/ethnic groups. By restricting the unsafe use of ESAs, medical costs associated with ESAs would decrease. The issue, however, is that some other medical costs (eg, costs of blood transfusions) might increase. It is critically important for CMS to understand the intended and potential unintended economic outcomes of a policy change when making policy decisions. It is also significant for CMS to understand whether the policy change had different effects on different sex and racial/ethnic groups.

To our knowledge, this study is the first to evaluate the economic consequences of the Medicare reimbursement policy change among ESA users. This study found that the Medicare reimbursement policy change of anemia-related costs (an 11\% reduction) and total medical costs (a 12\% reduction) in incident ESA users was similar. However, the impact of the policy change on different components of medical costs was different. For anemia-related costs, a greater cost saving was observed in patient cost sharing (an $18 \%$ reduction) than in Medicare payment (a 10\% reduction). In total medical costs, cost savings in patient cost sharing 


\section{TABLE 3 Change in Total Medical Costs Before and After Medicare Reimbursement Policy Change}

\begin{tabular}{|c|c|c|c|c|c|c|}
\hline & \multicolumn{2}{|c|}{ Total medical costs } & \multicolumn{2}{|c|}{$\begin{array}{l}\text { Medicare payment of total } \\
\text { medical costs }\end{array}$} & \multicolumn{2}{|c|}{$\begin{array}{l}\text { Patient cost sharing of total } \\
\text { medical costs }\end{array}$} \\
\hline & $\beta$ & $95 \% \mathrm{Cl}$ & $\beta$ & $95 \% \mathrm{Cl}$ & $\beta$ & $95 \% \mathrm{Cl}$ \\
\hline \multicolumn{7}{|l|}{ Policy change } \\
\hline Prepolicy period & Ref & & Ref & & Ref & \\
\hline Postpolicy period & 0.06 & $(0.01,0.12)$ & 0.07 & $(0.01,0.12)$ & 0.04 & $(-0.02,0.09)$ \\
\hline \multicolumn{7}{|l|}{ Group assignment } \\
\hline Control group & Ref & & Ref & & Ref & \\
\hline Treatment group & 0.19 & $(0.15,0.23)$ & 0.16 & $(0.12,0.20)$ & 0.37 & $(0.32,0.41)$ \\
\hline \multicolumn{7}{|l|}{ Policy change $\times$ group assignment } \\
\hline Otherwise & Ref & & Ref & & Ref & \\
\hline Postpolicy period and treatment group & -0.12 & $(-0.18,-0.06)$ & -0.11 & $(-0.18,-0.05)$ & -0.14 & $(-0.20,-0.07)$ \\
\hline \multicolumn{7}{|l|}{ Age, years } \\
\hline $66-69$ & Ref & & Ref & & Ref & \\
\hline $70-74$ & -0.04 & $(-0.07,-0.01)$ & -0.04 & $(-0.07,-0.01)$ & -0.04 & $(-0.07,-0.01)$ \\
\hline $75-79$ & -0.07 & $(-0.10,-0.04)$ & -0.06 & $(-0.09,-0.03)$ & -0.10 & $(-0.13,-0.07)$ \\
\hline $80+$ & -0.15 & $(-0.18,-0.12)$ & -0.14 & $(-0.18,-0.11)$ & -0.20 & $(-0.23,-0.17)$ \\
\hline \multicolumn{7}{|l|}{ Sex } \\
\hline Male & 0.06 & $(0.03,0.08)$ & 0.05 & $(0.03,0.07)$ & 0.07 & $(0.05,0.10)$ \\
\hline Female & Ref & & Ref & & Ref & \\
\hline \multicolumn{7}{|l|}{ Race/ethnicity } \\
\hline White & Ref & & Ref & & Ref & \\
\hline Black & 0.05 & $(0.01,0.10)$ & 0.06 & $(0.00,0.10)$ & 0.04 & $(0.00,0.08)$ \\
\hline Hispanic & 0.10 & $(0.00,0.19)$ & 0.10 & $(0.01,0.20)$ & 0.08 & $(-0.01,0.17)$ \\
\hline Other & 0.03 & $(-0.02,0.09)$ & 0.04 & $(-0.02,0.09)$ & 0.03 & $(-0.02,0.08)$ \\
\hline \multicolumn{7}{|l|}{ Urbanicity } \\
\hline Metropolitan & Ref & & Ref & & Ref & \\
\hline Nonmetropolitan & -0.06 & $(-0.09,-0.03)$ & -0.07 & $(-0.10,0.04)$ & 0.00 & $(-0.03,0.03)$ \\
\hline
\end{tabular}

(a $14 \%$ reduction) and Medicare payment (an $11 \%$ reduction) were similar.

We also found that the policy change was only effective in reducing medical costs in patients who were male and who were White. The policy change did not affect patients who were female, Black, Hispanic, or other races/ethnicities in terms of medical costs. Compared with patients who were female and of racial/ethnic minorities, patients who were male and who were White were less likely to use alternative strategies (eg, blood transfusion) to manage anemia when ESAs were not provided. ${ }^{20}$ Medical costs of alternative strategies and their complications might have offset the reduction in ESA costs.
This study found that patient cost sharing was reduced after the Medicare reimbursement policy change for patients who were male and female. Medical costs of cancer treatments were high and kept increasing. ${ }^{25}$ High patient cost sharing was found to be associated with a lower likelihood of initiating and adhering to cancer treatments. ${ }^{26,27}$

Study findings also revealed that evidence-based decision making could have contributed to the reduction in medical expenditures. In clinical practice, health care providers should actively engage patients in shared decision making for the selection of cost-effective cancer treatments. From a societal perspective, policymakers could use real-world evidence to make an informed decision 


\section{TABLE 3 Change in Total Medical Costs Before and After Medicare Reimbursement Policy Change (continued)}

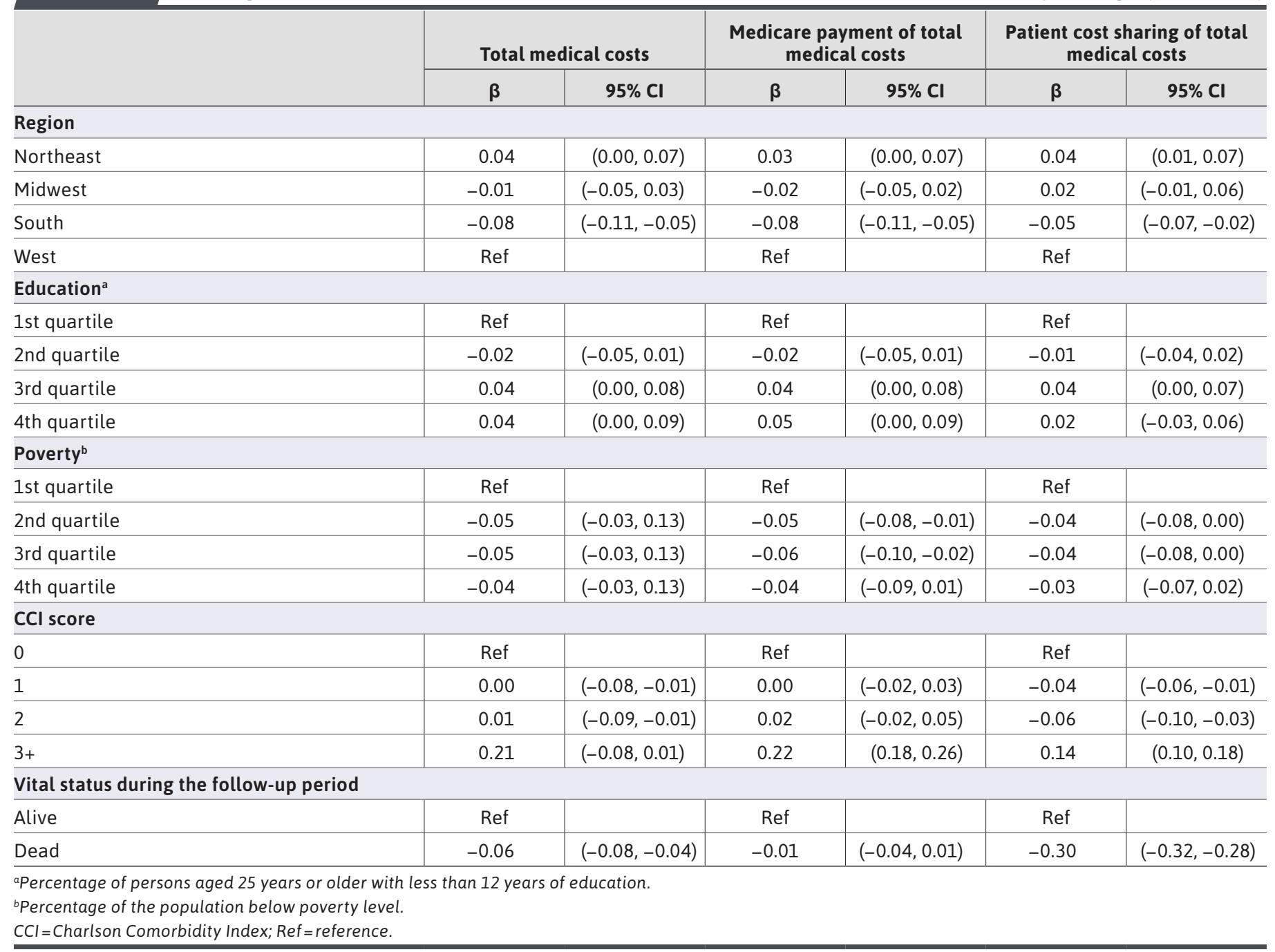

to improve health outcomes and reduce medical costs for cancer patients. The recent update of the ASCO/ASH clinical practice guidelines on the management of cancerassociated anemia with ESAs could be used to guide future evidence-based policy considerations. ${ }^{28}$

The findings of this study are of great significance not only for evaluating the impact of the Medicare reimbursement policy change but also for providing critical empirical evidence for CMS's future policy considerations. This study could inform CMS about medical costs of the Medicare reimbursement policy change by examining the difference in medical costs before and after the policy change. This information is of great significance to CMS because smart spending is one of its top objectives. As the highestexpenditure drug in the Medicare system, CMS identified new ways to pay for ESAs that can lower medical costs. This study also informs CMS to evaluate the effects of the policy change on females and racial/ethnic minorities. It is important to ensure that the voices and needs of minority populations are present when implementing new programs and policies. CMS should develop programs and policies to reduce health disparities and reach health equity.

\section{STRENGTHS AND LIMITATIONS}

The strengths of this study should be highlighted. First, to our knowledge, this study is the first to assess the impact 


\section{TABLE 4} Subgroup Analysis on Change in Anemia-Related and Total
Medical Costs Before and After Medicare Reimbursement
Policy Change by Sex and Race/Ethnicity

\begin{tabular}{l|c|c|c|c|c|c}
\hline \multirow{2}{*}{} & \multicolumn{2}{|c|}{ Sex } & \multicolumn{4}{c}{ Race/Ethnicity } \\
\cline { 2 - 7 } & Male & Female & White & Black & Hispanic & Other \\
\cline { 2 - 7 } & $\boldsymbol{\beta}$ & $\boldsymbol{\beta}$ & $\boldsymbol{\beta}$ & $\boldsymbol{\beta}$ & $\boldsymbol{\beta}$ & $\boldsymbol{\beta}$ \\
\hline Anemia-related costs & $-0.21^{\mathrm{a}}$ & -0.04 & $-0.16^{\mathrm{a}}$ & 0.12 & 0.27 & -0.06 \\
\hline Medicare payment & $-0.20^{\mathrm{a}}$ & -0.03 & $-0.15^{\mathrm{a}}$ & 0.13 & 0.34 & -0.05 \\
\hline Patient cost sharing & $-0.26^{\mathrm{a}}$ & $-0.13^{\mathrm{a}}$ & $-0.21^{\mathrm{a}}$ & 0.00 & -0.30 & -0.12 \\
\hline Total medical costs & $-0.18^{\mathrm{a}}$ & -0.08 & $-0.16^{\mathrm{a}}$ & 0.10 & -0.10 & -0.04 \\
\hline Medicare payment & $-0.18^{\mathrm{a}}$ & -0.07 & $-0.15^{\mathrm{a}}$ & 0.10 & -0.07 & -0.02 \\
\hline Patient cost sharing & $-0.18^{\mathrm{a}}$ & $-0.10^{\mathrm{a}}$ & $-0.16^{\mathrm{a}}$ & 0.09 & -0.29 & -0.14 \\
\hline aSignificant at 0.05 level. & \multicolumn{7}{|l}{} \\
\hline
\end{tabular}

of the Medicare reimbursement policy change on ESA costs and associated health disparities. CMS needs policy evaluation studies to assess if the ESA policy change generated cost savings among users with chemotherapyinduced anemia and reduced health disparities.

Second, a control group of patients with CKD was included in this study. Incorporating a control group eliminates multiple possible threats to internal validity (eg, the FDA's black box warning), thus, enhancing the internal validity of the study.

Third, a difference-in-difference design was used. The underlying assumption of using a simple pre-post design is that there is nothing associated with the outcome that happened at the same time as the policy change. The difference-in-difference design does not require such an assumption because it has a comparison group that is not subject to the policy change.

This study also has some limitations to consider. First, some potential confounding variables were not observed in the study. The SEER-Medicare linked data contain little information on physician characteristics and behavioral factors, thus, limiting our ability to measure and control for these potential confounding variables in the study. However, this study incorporated a control group, which enabled us to control for biases caused by confounding variables common to the treatment and control groups, even when they were unobserved.

Second, the impact of the policy change on medical costs in the long term was not examined. Some medical costs associated with ESA might have occurred 1 year after the index date. This study might not have been able to observe all medical costs associated with ESA during the 1-year follow-up period.

Third, this study examined medical costs of the Medicare reimbursement policy change in incident ESA users only. Medical costs were not examined in prevalent ESA users. Fourth, this study cannot measure ESA costs when paid fully by patients. Medicare claims data cannot measure medical expenditures not submitted for reimbursement. Fifth, CKD patients might not have been the perfect control group. They were not similar to the treatment group of cancer patients in all aspects.

Finally, this study was subject to spillover effects. When spillover occurred, physicians treating CKD patients might have changed their behaviors by reducing the ESA prescriptions based on the policy change for cancer patients. The spillover from the treatment group to the control group can lead to an underestimation of the true policy effect.

\section{Conclusions}

This study found that the Medicare ESA reimbursement policy change had an impact on the ESA-associated costs for cancer patients with chemotherapy-induced anemia. Anemia-related and total medical costs associated with ESAs in cancer patients with chemotherapy-induced anemia were reduced by about $10 \%$ after the Medicare reimbursement policy change. However, the policy change was only effective in male and White patients. The policy change had no effect on patients who were female and of racial/ethnic minorities.

This study provided real-world evidence of the economic consequence of the policy change in cancer patients with chemotherapy-induced anemia. ESAs were the highest-expenditure drug in Medicare Part B before the Medicare reimbursement policy change. This policy change was effective in lowering CMS expenditures for male and White patients.

\section{DISCLOSURES}

This study was funded by the SPARC Research Grant. The funder had no role in any part of this study. This study used the linked SEER-Medicare database. The interpretation and reporting of the data are the sole responsibility of the authors. The authors have nothing to disclose. 


\section{ACKNOWLEDGMENTS}

The authors acknowledge the efforts of the National Cancer Institute; the Centers for Medicare \& Medicaid Services Office of Research, Development and Information; Information Management Services, Inc.; and the Surveillance, Epidemiology, and End Results (SEER) Program tumor registries in the creation of the SEER-Medicare database.

\section{REFERENCES}

1. Knight K, Wade S, Balducci L.

Prevalence and outcomes of anemia in cancer: a systematic review of the literature. Am J Med. 2004;116(suppl 7A):11S-26S.

2. Groopman JE, Itri LM. Chemotherapyinduced anemia in adults: incidence and treatment. J Natl Cancer Inst. 1999;91(19):1616-34.

3. Rodgers GM 3rd, Becker PS, Blinder M, et al. Cancer- and chemotherapyinduced anemia. J Natl Compr Canc Netw. 2012;10(5):628-53.

4. Ludwig H, Fritz E. Anemia in cancer patients. Semin Oncol. 1998;25(3 suppl 7): 2-6.

5. Stauffer ME, Fan T. Prevalence of anemia in chronic kidney disease in the United States. PloS One. 2014;9(1):e84943.

6. Steinbrook R. Medicare and erythropoietin. N Engl J Med. 2007;356(1):4-6.

7. Rizzo JD, Lichtin AE, Woolf SH, et al. Use of epoetin in patients with cancer: evidence-based clinical practice guidelines of the American Society of Clinical Oncology and the American Society of Hematology. Blood. 2002;100(7):2303-20.

8. Rizzo JD, Somerfield MR, Hagerty KL, et al. Use of epoetin and darbepoetin in patients with cancer: 2007 American Society of Clinical Oncology/American Society of Hematology clinical practice guideline update. J Clin Oncol. 2008;26(1):132-49.

9. Rizzo JD, Brouwers M, Hurley P, et al. American Society of Clinical Oncology/ American Society of Hematology clinical practice guideline update on the use of epoetin and darbepoetin in adult patients with cancer. J Clin Oncol. 2010;28(33):4996-5010.
10. Arbuckle RB, Griffith NL, Iacovelli LM, et al. Continued challenges with the use of erythropoiesis-stimulating agents in patients with cancer: perspectives and issues on policy-guided health care. Pharmacotherapy. 2008;28(5 Pt 2):1S-15S.

11. Antos J. Medicare coverage policies for biologics: the broad gray line. Am Health Drug Benefits. 2008;1(3):13-20.

12. Sliver SM. Medicare coverage for erythropoiesis-stimulating agents: the perfect storm. Am Health Drug Benefits. 2008;1(4):46-50.

13. Sliver SM. Erythropoiesis-stimulating agents in a meta-stable state: guidelines, economics, and policy in flux. Am Health Drug Benefits. 2008;1(9):14-18.

14. Noxon V, Knopf KB, Norris LB, et al. Tale of two erythropoiesis-stimulating agents: utilization, dosing, litigation, and costs of darbepoetin and epoetin among South Carolina Medicaid-covered patients with cancer and chemotherapy-induced anemia. J Oncol Pract. 2017;13(6):e562-e573.

15. Bennett CL, McKoy JM, Henke M, et al Reassessments of ESAs for cancer treatment in the US and Europe. Oncology (Williston Park, NY). 2010;24(3):260-68.

16. Hess G, Nordyke RJ, Hill J, Hulnick S. Effect of reimbursement changes on erythropoiesis-stimulating agent utilization and transfusions. Am J Hematol. 2010;85(11):838-43.

17. Henry DH, Langer CJ, McKenzie RS, et al. Hematologic outcomes and blood utilization in cancer patients with chemotherapy-induced anemia (CIA) pre- and post-national coverage determination (NCD): results from a multicenter chart review. Support Care Cancer. 2012;20(9):2089-96.

18. Arneson TJ, Li S, Gilbertson DT, Bridges KR, Acquavella JF, Collins AJ. Impact of Centers for Medicare \& Medicaid Services national coverage determination on erythropoiesis-stimulating agent and transfusion use in chemotherapy-treated cancer patients. Pharmacoepidemiol Drug Saf. 2012;21(8):857-64.
19. Hershman DL, Neugut AI, Shim JJ, Glied S, Tsai WY, Wright JD.

Erythropoiesis-stimulating agent use after changes in medicare reimbursement policies. J Oncol Pract. 2014;10(4):264-69.

20. Li M, Schulz R, Chisholm-Burns M, Wang J, Lu ZK. Racial/ethnic and sex disparities in the use of erythropoiesisstimulating agents and blood transfusions: cancer management under Medicare's reimbursement policy. J Manag Care Spec Pharm. 2020;26(11):1477-86.

21. Steinbrook R. Medicare and erythropoietin. N Engl J Med. 2007;356(1):4-6.

22. KDOQI. KDOQI clinical practice guideline and clinical practice recommendations for anemia in chronic kidney disease: 2007 update of hemoglobin target. Am J Kidney Dis. 2007;50(3):471-530.

23. Dimick JB, Ryan AM. Methods for evaluating changes in health care policy: the difference-in-differences approach. JAMA 2014;312(22):2401-02.

24. Diehr P, Yanez D, Ash A, Hornbrook M, Lin DY. Methods for analyzing health care utilization and costs. Annu Rev Pub Health. 1999;20:125-44.

25. Mariotto AB, Yabroff KR, Shao Y, Feuer EJ, Brown ML. Projections of the cost of cancer care in the United States: 2010-2020. J Natl Cancer Inst. 2011;103(2):117-28.

26. Dusetzina SB, Winn AN, Abel GA, Huskamp HA, Keating NL. Cost sharing and adherence to tyrosine kinase inhibitors for patients with chronic myeloid leukemia. J Clin Oncol. 2014;32(4):306-11.

27. Streeter SB, Schwartzberg L, Husain N, Johnsrud M. Patient and plan characteristics affecting abandonment of oral oncolytic prescriptions. J Oncol Pract. 2011;7(3 suppl):46s-51s.

28. Bohlius J, Bohlke K, Castelli R, et al. Management of cancer-associated anemia with erythropoiesis-stimulating agents: ASCO/ASH clinical practice guideline update. J Clin Oncol. 2019;37(15):1336-51. 